\title{
Landau-like expansion for phase transitions in stochastic resetting
}

\author{
Arnab Pal ${ }^{1,2,3, *, \dagger}$ and V. V. Prasad ${ }^{4, *,+}$ \\ ${ }^{1}$ School of Chemistry, Raymond and Beverly Sackler Faculty of Exact Sciences, Tel Aviv University, Tel Aviv 6997801, Israel \\ ${ }^{2}$ Center for the Physics and Chemistry of Living Systems, Tel Aviv University, Tel Aviv 6997801, Israel \\ ${ }^{3}$ The Sackler Center for Computational Molecular and Materials Science, Tel Aviv University, Tel Aviv 6997801, Israel \\ ${ }^{4}$ Department of Physics of Complex Systems, Weizmann Institute of Science, Rehovot 7610001, Israel
}

(Received 25 April 2019; published 7 October 2019)

\begin{abstract}
We develop a Landau-like theory to characterize phase transitions in resetting systems. Restart can either accelerate or hinder the completion of a first passage process. The transition between these two states or phases is characterized by the behavioral change in the order parameter of the system namely the optimal restart rate which can undergo a first- or second-order transition depending on the details of the system. Nonetheless, there is no generic understanding of how the optimal restart rate behaves close to the transition with respect to the system parameters and what precisely characterizes the nature of the transition. We build a comprehensive theory to investigate both the transitions which, we show, can be understood by analyzing the first passage time moments. Our approach predicts the emergence of first- and second-order transition lines between the two phases that merge at a tricritical point. Power of our formulation is demonstrated on two canonical paradigm setup, namely, the Michaelis-Menten chemical reaction and diffusion under restart.
\end{abstract}

DOI: 10.1103/PhysRevResearch.1.032001

Consider a perpetual, nonequilibrium process governed by any law of motion that gets stopped intermittently and reinstated to a pre-selected configuration. The process resumes and continues until the next stopping epoch. This is known as the resetting or restart phenomena [1-32]. A fingerprint aspect of the topic that has gained considerable attention is its ramifications to any arbitrary first passage time process [33-36]. Indeed an underlying process that becomes subject to a constant restart rate can generically show different behavior since restart can either hinder or aid to its completion. In the former case, optimal restart rate (ORR) that minimizes the mean first passage time (MFPT) is fixed at zero while in the latter case one always obtains a nonzero restart rate which will minimize the MFPT and thus the ORR is finite. This means that by tuning the restart rate to this optimal value, the completion can be expedited. No surprise that this observation has taken the center stage and lead to a myriad of studies. In particular, restart renders mean first passage time finite for many diffusive $[1,2,25-27,29,30]$ and nondiffusive search processes $[18,21,28]$. Further studies revealed existence of the dominant restart strategy which will globally optimize the MFPT [19,20,23], and a genre of universality relations associated with the optimally restarted processes $[22,24]$.

Although most of the current investigations focused on whether restart overperforms or underperforms the

\footnotetext{
*Both authors contributed equally to this work.

$\dagger$ Corresponding author: arnabpal@ mail.tau.ac.il

prasad.vv@weizmann.ac.il
}

Published by the American Physical Society under the terms of the Creative Commons Attribution 4.0 International license. Further distribution of this work must maintain attribution to the author(s) and the published article's title, journal citation, and DOI. completion, sharp identification of the behavioral transition that accompanies this is quite less understood. We argue that this transition is in the same genre of canonical phase transition prevalent in equilibrium statistical physics and the ORR naturally plays the role of the order parameter. To be specific, a finite value of the ORR indicates that restart can speed-up the completion while a zero value of the same entails that restarting the process will leave unchanged or disfavor the completion. Restart transitions can be both first and second order like in the classical phase transition. In the former case, the ORR will have a discontinuous jump while in the latter case, it continuously vanishes to zero. First-order transition in ORR was observed in the case of a nondiffusive search with the aid of Lévy flight [17] or a search comprising of a drifted random walker with exponential flights subjected to restart [21]. The continuous transition in the restart rate, on the other hand, was observed in a system of Brownian walker in a domain or force field subject to resetting [25-27]. Although each variant above carried with it some unique and alluring features, there exists no unified framework which can describe the existence of both the transitions in a single system and other higher-order features such as a shift in transition from being continuous to first order. To bridge the gap, in this Rapid Communication, we systematically develop a Landau like theory which illustrates the rich structure in restart transitions and furthermore point out how the transitions are related to the criterion for the benefits of restart.

The phenomenological theory developed by Landau describes the universal behavior of a system near the phase transition [37-39]. According to this theory one can write down the free-energy of a system as a polynomial in the order parameters with the coefficients which play the key role in describing the transitions. Following Landau theory, we express the MFPT as a power series in terms of the restart rate $r$ near 
the transition, and show how by utilizing the relations between the coefficients, it is possible to predict the emergence of both first- and second-order transitions. We start by writing the MFPT under restart as a polynomial in restart rate

$$
\mathcal{T}(r)=a_{0}+a_{1} r+a_{2} r^{2}+a_{3} r^{3}+\cdots,
$$

where $a_{i}$ 's are the expansion coefficients. Such kind of expansion respects a set of postulates which we state below. By taking $r$ strictly to be zero, we see that $\mathcal{T}=a_{0}$ which is the MFPT without restart and is assumed to be positive finite. Notably when the underlying MFPT is infinite (e.g., diffusive search), introduction of any finite restart rate will always expedite the process and thus there will be no behavioral transition [23]. The expansion also assumes that $\mathcal{T}$ is an analytic function of both $r$ and the coefficients. Since $r$ is strictly a non-negative quantity, the system does not have any symmetry around $r=0$, and thus $\mathcal{T}$ can have all order terms in $r$. Importantly, the coefficient of the highest order in $r$ must be positive, otherwise one can minimize $\mathcal{T}$ by $r \rightarrow \infty$. We will now extensively explore how using the expansion as stated above one can capture the generic features of first- and second-order transitions.

First-order phase transition. First-order transition marks a discontinuous jump of the ORR from a finite value to zero at the transition point. This results as the absolute minimum of the MFPT switches between the two local minima at the transition. Landau's theory can be useful to explain the firstorder transition when the magnitude of this jump is small enough such that the expansion is justified. Here, we consider the MFPT upto the third-order term in the series such that $\mathcal{T}(r)=a_{0}+a_{1} r+a_{2} r^{2}+a_{3} r^{3}$, where $a_{1}>0$ and $a_{3}>0$. Given the expansion, the sign of $a_{2}$ decides whether there will be a first-order transition. If $a_{2} \geqslant 0$, the ORR is at zero since $\mathcal{T}$ monotonically increases as a function of restart rate. On the other hand when $a_{2}<0$, it is possible to have a finite ORR. The transition is then characterized by two simple relations

$$
\mathcal{T}\left(r_{f}\right)=\mathcal{T}(0),\left.\quad \frac{\partial \mathcal{T}}{\partial r}\right|_{r=r_{f}}=0,
$$

where $r_{f}$ is the ORR. From Eq. (2), we find $a_{1} r_{f}+a_{2} r_{f}^{2}+$ $a_{3} r_{f}^{3}=0$ and $a_{1}+2 a_{2} r_{f}+3 a_{3} r_{f}^{2}=0$. Solving these two equations, we obtain

$$
r_{f}=\frac{\left|a_{2}\right|}{2 a_{3}}, \quad a_{1}=\frac{a_{2}^{2}}{4 a_{3}}>0 .
$$

The locus of the ORR as a function of $a_{1}$ can be obtained by solving Eq. (2). This gives $r_{f}=\frac{\left|a_{2}\right|}{3 a_{3}}\left[1+\sqrt{1-3 a_{1} a_{3} / a_{2}^{2}}\right]>0$, which at the transition point $a_{1}=a_{2}^{2} / 4 a_{3}$, terminates at a value $\left|a_{2}\right| / 2 a_{3}$ and jumps discontinuously to zero. Evidently, the jump in ORR $\left(\Delta r_{f}\right)$ at the transition point can be captured by a parametric equation

$$
\Delta r_{f}=\left\{\begin{array}{ll}
0 & \text { if } a_{2} \geqslant 0 \\
\left|a_{2}\right| / 2 a_{3} & \text { if } a_{2}<0
\end{array} .\right.
$$

Second-order phase transition. The second-order phase transition is marked by the continuous vanishing of the ORR near the critical point. Prediction of this transition behavior has been carried out in specific setups which resorted to analyzing MFPT near $r \rightarrow 0$ [25-27]. We follow the same route and confer that indeed the transition is determined by the signchange in slope (i.e., $a_{1}$ ) of $\mathcal{T}$ near $r \rightarrow 0$ in Eq. (1) (truncated upto third order) where $a_{0} \geqslant 0$ and $a_{2}>0, a_{3}>0$. First we obtain the ORR $\left(r_{s}\right)$ by setting $\left.\frac{\partial \mathcal{T}}{\partial r}\right|_{r=r_{s}}=0$. The resulting equation yields a valid solution of the ORR, namely, $r_{s}=$ $\frac{a_{2}}{3 a_{3}}\left[\sqrt{1+3\left|a_{1}\right| a_{3} / a_{2}^{2}}-1\right]>0$, for $a_{1}<0$ [27]. Now as we tune the parameters such that $a_{1}$ changes sign from negative to positive, the ORR continuously shifts from $r_{s}>0$ to $r_{s}=0$. Close to the critical point, approach of ORR to zero maybe captured taking the limit $a_{1} \rightarrow 0$ which renders a linear behavior, i.e., $r_{s} \sim\left|a_{1}\right| / 2 a_{2}$. Thus ORR is found to follow a universal power law $r_{s} \propto\left|a_{1}\right|^{\beta}$, where $\beta=1$ [40]. Notably, this relation is reminiscent of the same in order parameter observed, e.g., in liquid-gas or ferromagnetic systems where $\beta$ is found to be universal.

Treating the first-order transitions using Landau's expansion reveals a significant caveat. If the first-order transition involves a discontinuity in ORR from zero to a finite value significantly away from zero, it cannot be captured by a small order expansion of $\mathcal{T}$ near $r=0$. In fact, in this case, one may have to include the higher-order terms in the power series expansion depending on the specific problem. It is clear, however, that this expansion becomes more respectable as the discontinuity $\left|a_{2}\right| / 2 a_{3}$ in the order parameter becomes smaller, particularly when it goes to zero. Thus the theory is expected to describe well when

$$
a_{1}=0, \quad a_{2}=0,
$$

which is the tricritical point. In a phase diagram spanned by the system parameters, Eq. (5) denotes the tricritical point where the first- and second-order transition lines merge [41].

Physical meaning of the coefficients. The coefficients $a_{i}$ 's, so far defined formally, can be given physical meaning by analyzing $\mathcal{T}$ around $r=0$. MFPT for a generic process under constant restart rate $r$ is given by $\mathcal{T}=\frac{1-\tilde{T}(r)}{r \tilde{T}(r)}$, where $\tilde{T}(r)=\left\langle e^{-r T}\right\rangle$ is the Laplace transformation of the underlying first passage time, denoted by $T$, evaluated at $r$ [23]. Then, comparing the Taylor's series expansion of $\mathcal{T}$ to Eq. (1), we identify $a_{0}=\langle T\rangle, a_{1}=-\frac{\left\langle T^{2}\right\rangle}{2}+\langle T\rangle^{2}, a_{2}=$ $\frac{1}{6}\left\langle T^{3}\right\rangle+\langle T\rangle^{3}-\langle T\rangle\left\langle T^{2}\right\rangle, \quad a_{3}=-\frac{\left\langle T^{4}\right\rangle}{4 !}+\frac{\left\langle T^{3}\right\rangle\langle T\rangle}{3}+\frac{\left\langle T^{2}\right\rangle^{2}}{4}-$ $\frac{3\left\langle T^{2}\right\rangle\langle T\rangle^{2}}{2}+\langle T\rangle^{4}$, and so on where $\left\langle T^{n}\right\rangle$ is the $n$th moment of the underlying first passage time distribution [41]. The moments (hence the coefficients) are explicit functions of the system parameters, and will characterize the transitions. Recall that the second-order phase transition is characterized by the change in sign of $a_{1}$ which would imply whether the coefficient of variation $(\mathrm{CV})$, which stands for the ratio between the standard deviation and the mean of $T$, is higher or lower than unity [23]. We find that this remains as a sufficient condition for the second-order transition but not for the first order where the sign of $a_{2}$ plays the pivotal role. Interestingly, this immediately makes a connection with the existing criterion for restart to help improve a process [23]. Although $\mathrm{CV}>1$ (i.e., $a_{1}<0$ ) still remains as a sufficient condition for restart to help, it may not be necessary. In fact, 
(a)

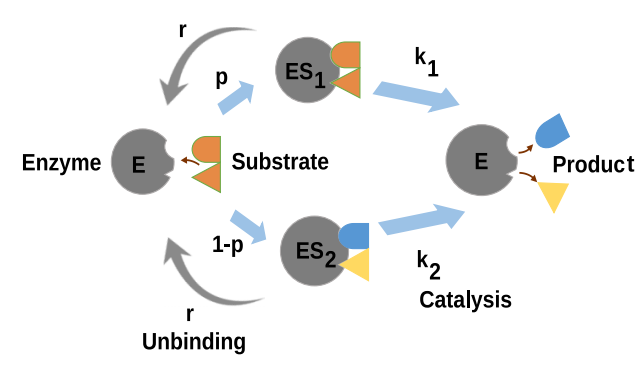

(b)

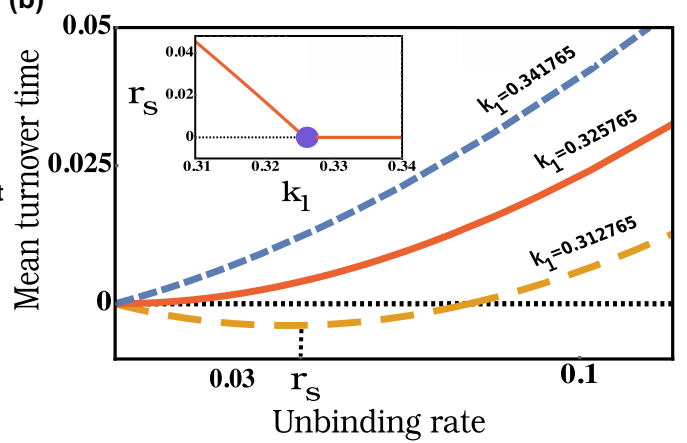

FIG. 1. (a) Illustration of a two-state Michaelis-Menten chemical reaction. (b) Shifted $\mathcal{T}$ as a function of the unbinding rate $r$ for $k_{2}=1, p=1 / 3$ for different $k_{1}$ (values marked on the plots). The minima $r_{s}$ (shown by the dashed vertical line) corresponds to the ORR which continuously vanishes to zero at the critical point $k_{1}=0.3258$ (in blue circle) as shown in the inset.

even when $\mathrm{CV}<1$ (i.e., $a_{1}>0$ ), first-order transition can occur (due to the change in sign of $a_{2}$ ) and restart would still be beneficial (see examples). To illustrate the frame-work developed above, we now investigate two case studies, namely, the Michaelis-Menten reaction and diffusion under restart.

Michaelis-Menten chemical reaction. Michaelis-Menten chemical reactions are an integral part of first passage under restart (FPUR) formalism [42-46]. Here we will assume a two-state model of this reaction where an enzyme (E) binds with a substrate $(\mathrm{S})$ to form two alternative metastable states: $\mathrm{ES}_{1}$ with probability $p$ or $\mathrm{ES}_{2}$ otherwise [see Fig. 1(a)]. Either of the complexes can subsequently be converted into product (P) via catalysis which marks the turnover of an enzymatic reaction cycle (completion) or the enzyme can unbind (restart the reaction). Catalysis in each one of the branches is assumed to be an Erlang process and differs between the two states through their effective catalytic rates given by $k_{1}$ and $k_{2}$. The effective time distribution for the resultant catalytic process will then have the form $f_{T_{\text {cat }}}(t)=p k_{1}^{2} t e^{-k_{1} t}+(1-$ p) $k_{2}^{2} t e^{-k_{2} t}$, which is also known as the hyper-Erlang distribution [42-44]. The unbinding, however, is assumed to occur at a same constant rate $r$ from $\mathrm{ES}_{1}$ or $\mathrm{ES}_{2}$. Borrowing tools from the theory of FPUR, one immediately obtains the mean turnover time for the completion of a reaction cycle [41]

$$
\mathcal{T}=\frac{\left(k_{1}+r\right)^{2}\left[\left(k_{2}+r\right)^{2}-(1-p) k_{2}^{2}\right]-p k_{1}^{2}\left(k_{2}+r\right)^{2}}{r\left[p k_{1}^{2}\left(k_{2}+r\right)^{2}+(1-p) k_{2}^{2}\left(k_{1}+r\right)^{2}\right]}
$$

Expanding $\mathcal{T}$ around $r=0$ as in Eq. (1), one can immediately identify $a_{0}=2\left[k_{1}+\left(k_{2}-k_{1}\right) p\right] / k_{1} k_{2}, a_{1}=\left[3 k_{1}^{2}(-1+p)-\right.$ $\left.3 k_{2}^{2} p+4\left(k_{1}+\left(k_{2}-k_{1}\right) p\right)^{2}\right] /\left(k_{1} k_{2}\right)^{2}$, and others we reserve in Ref. [41]. The second-order transition is determined by $a_{1}=0$ which implies $3 k_{1}^{2}(-1+p)-3 k_{2}^{2} p+4\left(k_{1}+\left(k_{2}-\right.\right.$ $\left.\left.k_{1}\right) p\right)^{2}=0$. For fixed $k_{2}$ and $p$, the above equation gives a solution for $k_{1}$. To see the transition, we plot the mean turnover time (shifted by $\langle T\rangle$ ) as a function of the unbinding rate $r$ while varying the parameter $k_{1}$ (values marked in the plot) for $k_{2}=1, p=1 / 3$ in Fig. 1(b). As we vary $k_{1}$, the ORR $r_{s}$ continuously vanishes to zero from a finite value marking second-order transition. The transition occurs at $k_{1}=0.3258$ [blue marker in Fig. 1(b) inset], which can also be found by solving the above equation for the parameter values $k_{2}=$ $1, p=1 / 3$.

We now turn our attention to Fig. 2(a) where we have plotted shifted $\mathcal{T}$ as a function of $r$ for different $k_{1}$ fixing $k_{2}=1.0$ and $p=0.5002$. As we vary $k_{1}$, we observe that the ORR jumps from a finite value $r_{f}=3 \times 10^{-4}$ to zero and this transition occurs at $k_{1} \approx 0.267842 \ldots$ These values [indicated by markers in Fig. 2(a) inset] match exactly with the theoretical predictions. Note that although $a_{1}>0$ (i.e., $\mathrm{CV}<1$ ) in this case, restart still remains beneficial [see the orange dashed curve in Fig. 2(a)].

However, choice of the parameter co-ordinates used above is not merely coincidental. In fact, these are chosen at the proximity of the tricritical point which is determined by setting $a_{1}=0, a_{2}=0$. The second-order line in $\left(p, k_{1}\right)$ plane can be determined by $a_{1}=0$ for each fixed $k_{2}$. This is shown in solid lines in Fig. 2(b). In contrast, to generate the first-order contour line, one needs to consider the exact mean turnover time [Eq. (6)] and apply the conditions given by Eq. (2) [41]. We show these lines by dashed curves in Fig. 2(b). The firstand second-order lines meet at the tricritical points which are given by $p=\frac{1}{2}, k_{1}=k_{2}(2 \pm \sqrt{3})$. Therefore, for each $k_{2}$, there are two tricritical points in $\left(p, k_{1}\right)$ plane which are denoted by $\left(p_{a}^{T}, k_{1 a}^{T}\right)$ and $\left(p_{b}^{T}, k_{1 b}^{T}\right)$ [41] and in Fig. 2(a), we chose our coordinates near the second tricritical point. The phase diagram comprising first- and second-order transitions in $\left(p, k_{1}\right)$ space is illustrated in Fig. 2(b).

Diffusion under restart. We consider motion of a drifted Brownian particle (diffusion constant $D$ ) confined in a onedimensional domain with absorbing boundaries at $[0, L]$. Starting from $x_{0}$ at time zero, the particle is drifted towards the right boundary $L$ with velocity $v>0$. In addition, the particle is also reset back to $x_{0}$ with a constant rate $r$. The MFPT for the particle to escape through any of the boundaries is then found to be [41]

$$
\mathcal{T}=\frac{L^{2}}{D \mathrm{Pe}^{2}} \mathcal{G}(y, u, \mathrm{Pe})
$$

where $u=\frac{x_{0}}{L}$ and $\mathrm{Pe}=\frac{L v}{2 D}$ is the Péclet number. Along with $y \equiv \sqrt{1+4 D r / v^{2}}$, a dimensionless measure of the scaled 
(a)

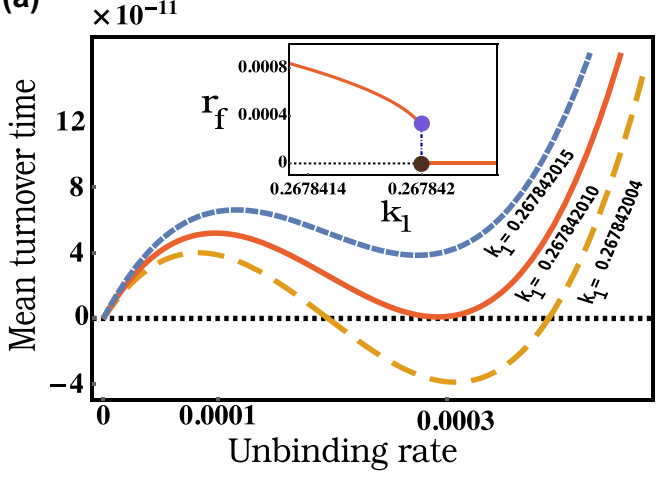

(b)

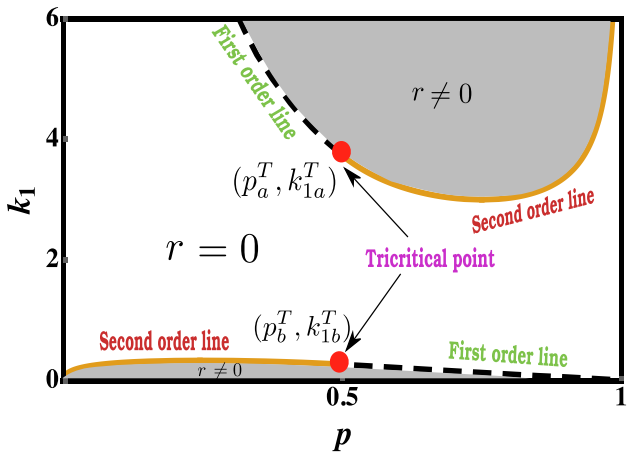

FIG. 2. (a) Shifted $\mathcal{T}$ as a function of $r$ for various $k_{1}$ fixing $k_{2}=1$ and $p=0.5002$. The ORR $r_{f}$ exhibits a discontinuous transition at $k_{1} \approx 0.26784201$ (in black circle) as shown in the inset. (b) Phase diagram in the $\left(p, k_{1}\right)$ plane for $k_{2}=1$. The shaded and nonshaded regions indicate the domain in the parameter space where ORR is positive and zero, respectively. The first- (in black dashed) and second- (in orange solid) order lines merge at the tricritical points marked by the red circles.

restart rate, the scaling function $\mathcal{G}$ reads

$$
\begin{aligned}
\mathcal{G} & (y, u, \mathrm{Pe}) \\
& =\frac{1}{y^{2}-1}\left(\frac{e^{u \mathrm{Pe}} \sinh [y \mathrm{Pe}]}{\sinh [(1-u) y \mathrm{Pe}]+e^{\mathrm{Pe}} \sinh [u y \mathrm{Pe}]}-1\right) .
\end{aligned}
$$

To get the ORR, we do the following optimization $\partial_{y} \mathcal{G}(y, u, \mathrm{Pe})=0$. Expanding $\mathcal{G}$ around $y=1$ (i.e., $r \rightarrow 0$ ) immediately reveals the coefficients $\left(a_{i} / \mathrm{s}\right)$ which we present in Ref. [41]. In particular note that the underlying MFPT $\langle T\rangle=$ $a_{0}$ is finite due to the choice of the finite geometry and thus in accordance with the expansion in Eq. (1). Setting $a_{1}=0$ we obtain a relation between Pe and $u$ that determines the secondorder critical point. To see the transition, we plot the ORR as a function of $u$ while fixing $\mathrm{Pe}=1$ in Fig. 3(a). This shows that $y_{s}$ continuously vanishes to zero at $u=0.6903$ [blue marker in Fig. 3(a)] as predicted from the theory. Note that if $u>0.6903$, we always find $y_{s}>1$, i.e., restart is beneficial; otherwise it will be detrimental. The linear scaling of the ORR $y_{s}$ with $u$ close to the critical point in Fig. 3(a) accords with the theory.

The setup also exhibits a rich first-order transition phenomena. To capture this transition, we once again examine the coordinates near the tricritical point $u^{T}=0.25186, \mathrm{Pe}^{T}=$ 0.43764 (marked by red circle in Fig. 4) which can be (a)

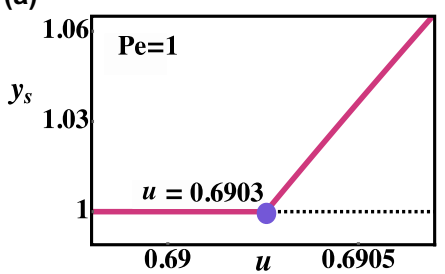

(b)

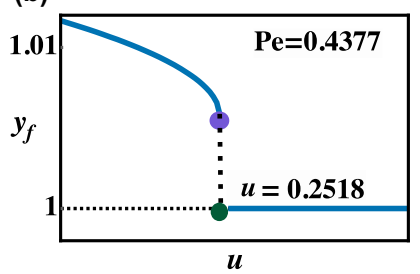

FIG. 3. (a) Continuous transition of ORR $y_{s}$ as a function of $u$ for a fixed $\mathrm{Pe}=1.0$. The transition occurs at the critical value $u=$ 0.6903 (in blue circle). (b) First-order phase transition in ORR $y_{f}$ as a function of $u$ for a fixed $\mathrm{Pe}=0.4377$. The ORR jumps from a finite value 1.0086 (in blue circle) to unity at the transition point $u=0.2518$ (in green circle). computed using Eq. (5). In Fig. 4, we have plotted the ORR as a function of $u$ and Pe. We see that for $u<u^{T}$ and $\mathrm{Pe}>\mathrm{Pe}^{T}$, the system undergoes a series of first-order transitions while the complementary limits show transitions of second order. Choosing $u=0.2518$ and $\mathrm{Pe}=0.4377$, we find that the ORR $y_{f}$ jumps from the value 1.0086 to unity discontinuously [Fig. 3(b)]. The value of the ORR is commensurate with the theoretical predictions given by Eq. (3). Although our framework does not capture the generic behavior of the firstorder transitions away from the tricritical point, one can still apprehend the entire phase diagram in the $(\mathrm{Pe}, u)$ parameter space (see inset of Fig. 4) by performing the same analysis as was done in the earlier case study [41].

Conclusions. Landau's theory of free energy expansion is a benchmark paradigm in equilibrium statistical physics. We show that a similar expansion of the MFPT as a function of restart rate serves as a natural platform to study the phase

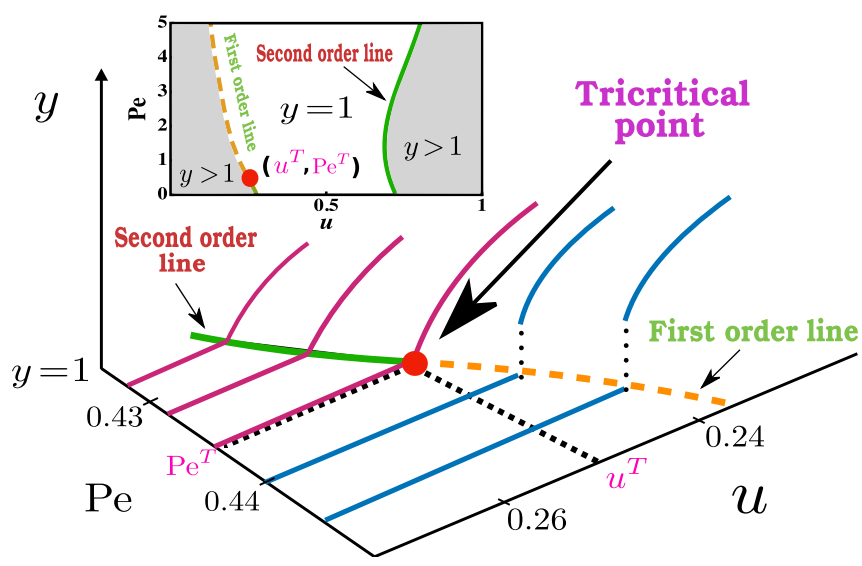

FIG. 4. Phase transitions in ORR in a drift-diffusion process. We plot the ORR as a function of $\mathrm{Pe}$ and $u$ close to the tricritical point (in red circle). The first- (in dashed orange) and second-order (in solid green) lines meet at the tricritical point: $u^{T}=0.25186$ and $\mathrm{Pe}^{T}=$ 0.43764. An extended phase diagram for the entire range of $u$ is depicted in the inset. The shaded regions represent domains with $y>1$, and the nonshaded one with $y=1$. At large Pe, the continuous transitions occur close to $u=1$ while the first-order transition is seen strictly near $u=0$. See Ref. [41] for further illustrations. 
transitions in ORR and crossover between the phases can entirely be characterized by the underlying process. Our theory describes elaborately the nature of the transitions and the critical points. As described before, higher-order critical points (such as the tricritical point) carry interesting topological signatures in a phase diagram (as shown in Figs. 2 and 4), which tend to be independent of the parameters. Thus the prediction of higher-order critical points has immense value in defining the nature of phase diagram. The formalism presented in the manuscript precisely does that, for a generic resetting system.

Furthermore, our analysis reveals that the well known criterion (for restart to be beneficial) in terms of the coefficient of variation $(\mathrm{CV})$ remains as a sufficient condition for the continuous phase transition but not the first order. In fact, the latter can take place even if the criterion is not satisfied which is a robust feature of the transition. Clearly, understanding this behavior is important in many systems, as it helps us predict whether resetting is advantageous or not to facilitate the completion of a process (see, e.g., Ref. [47], where restart has been engineered as a tool in an Anilin-promoted cell contraction). We conclude our discussion by stating that an extension of the theory to address transitions between two nonzero ORRs (as in Lévy search [17]) is yet to be addressed, and opts for a potential future problem.

Acknowledgments. Arnab Pal gratefully acknowledges support from the Raymond and Beverly Sackler Post-Doctoral Scholarship. We thank David Mukamel for fruitful discussions. We thank an anonymous reviewer for many insightful remarks.
[1] M. R. Evans and S. N. Majumdar, Diffusion with Stochastic Resetting, Phys. Rev. Lett. 106, 160601 (2011).

[2] M. R. Evans and S. N. Majumdar, Diffusion with optimal resetting, J. Phys. A: Math. Theor. 44, 435001 (2011).

[3] M. R. Evans, S. N. Majumdar, and K. Mallick, Optimal diffusive search: nonequilibrium resetting versus equilibrium dynamics, J. Phys. A: Math. Theor. 46, 185001 (2013).

[4] S. Gupta, S. N. Majumdar, and G. Schehr, Fluctuating Interfaces Subject to Stochastic Resetting, Phys. Rev. Lett. 112, 220601 (2014).

[5] A. Pal, Diffusion in a potential landscape with stochastic resetting, Phys. Rev. E 91, 012113 (2015).

[6] S. Eule and J. J. Metzger, Non-equilibrium steady states of stochastic processes with intermittent resetting, New J. Phys. 18, 033006 (2016).

[7] X. Durang, M. Henkel, and H. Park, The statistical mechanics of the coagulation-diffusion process with a stochastic reset, J. Phys. A: Math. Theor. 47, 045002 (2014).

[8] S. N. Majumdar, S. Sabhapandit, and G. Schehr, Dynamical transition in the temporal relaxation of stochastic processes under resetting, Phys. Rev. E 91, 052131 (2015).

[9] V. Méndez and D. Campos, Characterization of stationary states in random walks with stochastic resetting, Phys. Rev. E 93, 022106 (2016).

[10] A. Falcón-Cortés, D. Boyer, L. Giuggioli, and S. N. Majumdar, Localization Transition Induced by Learning in Random Searches, Phys. Rev. Lett. 119, 140603 (2017).

[11] R. Falcao and M. R. Evans, Interacting Brownian motion with resetting, J. Stat. Mech. (2017) 023204.

[12] S. N. Majumdar, S. Sabhapandit, and G. Schehr, Random walk with random resetting to the maximum position, Phys. Rev. E 92, 052126 (2015).

[13] A. Pal, R. Chatterjee, S. Reuveni, and A. Kundu, Local time of diffusion with stochastic resetting, J. Phys. A: Math. Theor. 52, 264002 (2019).

[14] M. R. Evans and S. N. Majumdar, Effects of refractory period on stochastic resetting, J. Phys. A: Math. Theor. 52, 01LT01 (2019).

[15] A. Pal, A. Kundu, and M. R. Evans, Diffusion under timedependent resetting, J. Phys. A: Math. Theor. 49, 225001 (2016).
[16] A. Nagar and S. Gupta, Diffusion with stochastic resetting at power-law times, Phys. Rev. E 93, 060102(R) (2016).

[17] L. Kusmierz, S. N. Majumdar, S. Sabhapandit, and G. Schehr, First Order Transition for the Optimal Search Time of Lévy Flights with Resetting, Phys. Rev. Lett. 113, 220602 (2014).

[18] L. Kusmierz and E. Gudowska-Nowak, Optimal first-arrival times in Lévy flights with resetting, Phys. Rev. E 92, 052127 (2015).

[19] U. Bhat, C. De Bacco, and S. Redner, Stochastic search with Poisson, and deterministic resetting, J. Stat. Mech. (2016) 083401.

[20] A. Chechkin and I. M. Sokolov, Random Search with Resetting: A Unified Renewal Approach, Phys. Rev. Lett. 121, 050601 (2018).

[21] D. Campos and V. Méndez, Phase transitions in optimal search times: How random walkers should combine resetting, and flight scales, Phys. Rev. E 92, 062115 (2015).

[22] S. Reuveni, Optimal Stochastic Restart Renders Fluctuations in First Passage Times Universal, Phys. Rev. Lett. 116, 170601 (2016).

[23] A. Pal and S. Reuveni, First Passage under Restart, Phys. Rev. Lett. 118, 030603 (2017).

[24] S. Belan, Restart Could Optimize the Probability of Success in a Bernoulli Trial, Phys. Rev. Lett. 120, 080601 (2018).

[25] C. Christou and A. Schadschneider, Diffusion with resetting in bounded domains, J. Phys. A: Math. Theor. 48, 285003 (2015).

[26] S. Ray, D. Mondal, and S. Reuveni, Péclet number governs transition to acceleratory restart in drift-diffusion, J. Phys. A: Math. Theor. 52, 255002 (2019).

[27] S. Ahmad, I. Nayak, A. Bansal, A. Nandi, and D. Das, First passage of a particle in a potential under stochastic resetting: A vanishing transition of optimal resetting rate, Phys. Rev. E 99 , 022130 (2019).

[28] A. Pal, I. Eliazar, and S. Reuveni, First Passage Under Restart with Branching, Phys. Rev. Lett. 122, 020602 (2019).

[29] A. Pal and V. V. Prasad, First passage under stochastic resetting in an interval, Phys. Rev. E 99, 032123 (2019).

[30] A. Pal, Ł. Kusmierz, and S. Reuveni, Home-range search provides advantage under high uncertainty, arXiv:1906.06987.

[31] A. Pal and S. Rahav, Integral fluctuation theorems for stochastic resetting systems, Phys. Rev. E 96, 062135 (2017). 
[32] U. Basu, A. Kundu, and A. Pal, Symmetric exclusion process under stochastic resetting, Phys. Rev. E 100, 032136 (2019).

[33] S. Redner, A Guide to First-Passage Processes. A Guide to FirstPassage Processes (Cambridge University Press, Cambridge, UK, 2007).

[34] R. Metzler, S. Redner, and G. Oshanin, First-Passage Phenomena, and Their Applications (World Scientific, Singapore, 2014), Vol. 35.

[35] A. J. Bray, S. N. Majumdar, and G. Schehr, Persistence, and first-passage properties in nonequilibrium systems, Adv. Phys. 62, 225 (2013).

[36] O. Bénichou, C. Loverdo, M. Moreau, and R. Voituriez, Intermittent search strategies, Rev. Mod. Phys. 83, 81 (2011).

[37] L. D. Landau and E. M. Lifshitz, Statistical Physics, Part 1, Course of Theoretical Physics (Elsevier ButterworthHeinemann, Oxford, UK, 1980), Vol. 5, p. 2.

[38] N. Goldenfeld, Lectures on Phase Transitions, and the Renormalization Group (CRC, Taylor and Francis Group, Boca Raton, FL, 2018).

[39] P. M. Chaikin, T. C. Lubensky, and T. A. Witten, Principles of Condensed Matter Physics (Cambridge University Press, Cambridge, 1995).

[40] It is possible to expand $a_{1}$ in terms of the control parameter $\lambda$ of the system (e.g., in the first example $\lambda=k_{1}$ ) in the $a_{1} \rightarrow 0$ limit (which corresponds to $\lambda$ reaching the critical point $\lambda^{*}$ ) under mild regularity conditions. This gives $a_{1}(\lambda)=a_{1}\left(\lambda^{*}\right)+$ $a_{1}^{\prime}\left(\lambda^{*}\right)\left(\lambda-\lambda^{*}\right)+\cdots$ Since $a_{1}\left(\lambda^{*}\right)=0$, the limit $a_{1} \rightarrow 0$ will then correspond to $r_{s} \propto\left|\lambda-\lambda^{*}\right|$ with $\beta=1$. The linear dependence of the ORR as a function of the control parameter near the critical point can be seen in Figs. 1(b) and 3(a).

[41] See Supplemental Material at http://link.aps.org/supplemental/ 10.1103/PhysRevResearch.1.032001 for a graphical illustration of phase transitions in resetting system, a detailed derivation of Eqs. (6) and (7), evaluation of coefficients and detailed analysis of phase diagram for the Michaelis-Menten set up and diffusion under restart, and depiction of first and second order transitions in ORR for diffusion under restart.

[42] S. Reuveni, M. Urbakh, and J. Klafter, Role of substrate unbinding in Michaelis-Menten enzymatic reactions, Proc. Natl. Acad. Sci. USA 111, 4391 (2014).

[43] T. Rotbart, S. Reuveni, and M. Urbakh, Michaelis-Menten reaction scheme as a unified approach towards the optimal restart problem, Phys. Rev. E 92, 060101(R) (2015).

[44] T. Robin, S. Reuveni, and M. Urbakh, Single-molecule theory of enzymatic inhibition, Nat. Commun. 9, 779 (2018).

[45] É. Roldán, A. Lisica, D. Sánchez-Taltavull, and S. W. Grill, Stochastic resetting in backtrack recovery by RNA polymerases, Phys. Rev. E 93, 062411 (2016).

[46] G. J. Lapeyre and M. Dentz, Reaction diffusion with stochastic decay rates, Phys. Chem. Chem. Phys. 19, 18863 (2017).

[47] S. Budnar, K. B. Husain, G. A. Gomez, M. Naghibosadat, A. Varma, S. Verma, N. A. Hamilton, R. G. Morris, and A. S. Yap, Anillin promotes cell contractility by cyclic resetting of RhoA residence kinetics, Developmental Cell 49, 894 (2019). 